\title{
Prevention of Symptomatic Vasospasm After Aneurysmal Subarachnoid Hemorrhage by Intraoperative Cisternal Fibrinolysis Using Tissue-Type Plasminogen Activator Combined With Continuous Cisternal Drainage
}

\author{
Hiroyuki KInOUCHI ${ }^{* * *}$, Kuniaki OGASAWARA**, Hiroaki SHIMIZU**, \\ Kazuo Mizor*, and Takashi Yoshimoto*** \\ *Department of Neurosurgery, Akita University School of Medicine, Akita; \\ ${ }^{* *}$ Department of Neurosurgery, Kohnan Hospital, Sendai; \\ *** Department of Neurosurgery, Tohoku University School of Medicine, Sendai
}

\begin{abstract}
The efficacy of intraoperative cisternal irrigation using tissue-type plasminogen activator (tPA) combined with continuous cisternal drainage was assessed for the prevention of symptomatic vasospasm in patients with aneurysmal subarachnoid hemorrhage (SAH). Seventy consecutive patients underwent direct surgery for aneurysm clipping within 48 hours of $\mathrm{SAH}$ and had computed tomography (CT) findings classified as Fisher group III or IV with densities of more than 65 Hounsfield units (HU). Fibrinolysis of the cisternal clots was performed during surgery using $1.6 \mathrm{mg}$ tPA in 55 cases or $3.2 \mathrm{mg}$ tPA in 15 cases. If postoperative CT within 24 hours of surgery showed areas with density more than 65 HU, additional tPA $(0.8 \mathrm{mg} / \mathrm{day})$ was administered into the cisternal catheter until the high density areas disappeared. The cisternal drainage catheters were left in place until day 14 . Additional tPA injection was necessary in four of the 55 patients receiving $1.6 \mathrm{mg}$ tPA. Symptomatic vasospasm occurred in three patients $(\mathbf{4 . 3 \%})$ and two patients had low density areas on CT. Permanent deficit (hemiparesis) due to cerebral vasospasm remained in only one patient. Intraoperative cisternal irrigation with tPA combined with cisternal drainage is safe and effective for the prevention of symptomatic vasospasm following SAH.
\end{abstract}

Key words: aneurysm, cisternal drainage, fibrinolysis, subarachnoid hemorrhage,

tissue-type plasminogen activator, vasospasm

\section{Introduction}

Development of severe vasospasm is generally accepted to be highly correlated with the amount and distribution of subarachnoid clots, ${ }^{8,27)}$ so evacuation of subarachnoid blood clots at an early stage is effective for preventing cerebral vasospasm..$^{9,14,17,26,28-31)}$ Subarachnoid clots cannot always be removed by surgical aspiration for technical reasons, so adjunct therapies to dissolve the clots using urokinase or tissue-type plasminogen activator (tPA) have been applied. ${ }^{2,6,15,16,18-20,23,24,32-34)}$ Vasospasm following aneurysmal subarachnoid hemorrhage $(\mathrm{SAH})$ can be prevented by the postoperative administration of tPA through catheters into the cisterns and ventricles. ${ }^{15,16)}$ However, the procedure of tPA injection through catheters for several days is relatively hazardous in daily clinical practice and carries a risk of complications such as infection and hematoma. ${ }^{16,21)}$

The present prospective study investigated the efficacy of a single intraoperative cisternal irrigation using tPA combined with continuous cisternal drainage to prevent symptomatic vasospasm in patients with aneurysmal SAH.

Received November 17, 2003; Accepted June 14, 2004

Author's present address: K. Ogasawara, M.D., Department of Neurosurgery, Iwate Medical University School of Medicine, Morioka, Japan. 


\section{Patients and Methods}

\section{Patient population}

This prospective study included 70 consecutive patients, 16 men and 54 women, aged between 13 and 82 years (mean 57 years), treated for ruptured cerebral aneurysm between January 1996 and July 1997 at Kohnan Hospital, Sendai, Japan. All patients underwent direct surgery within 48 hours and had computed tomography (CT) findings classified as Fisher group III (66 cases) or IV (4 cases) with densities of more than 65 Hounsfield units (HU). ${ }^{8)}$ The CT density of the subarachnoid clots was measured by region of interest analysis. ${ }^{16)}$ The aneurysms were located on the middle cerebral artery in 24 patients, the anterior communicating artery in 18 , the internal carotid artery in 14 , the vertebrobasilar artery (basilar tip or basilar artery-superior cerebellar artery junction) in six, and other sites in eight.

\section{Protocol for tPA treatment}

Intraoperative cisternal fibrinolysis was performed by bolus injection of a dose (1.6 or $3.2 \mathrm{mg}$ ) of tPA to the exposed cisterns. If postoperative CT within 24 hours of surgery showed areas with density more than $65 \mathrm{HU}$, additional cisternal injection of tPA ( $0.8 \mathrm{mg} /$ day) was performed until the high density areas disappeared. The catheters were clamped for 1 hour after administration to prevent the immediate outflow of the tPA. The drainage catheters were usually not removed at the completion of tPA administration, but were left in place for a period of 2 weeks. The tPA treatment protocol was based on the Japanese Ministry of Health guidelines and was accepted by the Clinical Research Board of the Kohnan Hospital.

\section{Surgical procedure}

Eight patients underwent bifrontal craniotomy (5 for anterior communicating artery aneurysm and 3 for distal anterior cerebral artery aneurysm) and the other patients underwent unilateral craniotomy. Patients with hydrocephalus underwent continuous ventricular drainage from the ipsilateral anterior horn of the lateral ventricle. Two patients underwent unilateral craniotomy for basilar tip aneurysm via the subtemporal approach. The other 60 patients were treated via the transsylvian approach. After clipping of the ruptured aneurysm, the ipsilateral sylvian fissure, carotid cistern, prechiasmatic cistern, Liliequist's membrane, and the contralateral proximal sylvian fissure were opened. tPA $1.2 \mathrm{mg}$ or $2.8 \mathrm{mg}$ in $0.08 \mathrm{mg} / \mathrm{ml}$ of saline) was then injected into the cisterns. The clots were allowed to dissolve for more than 30 minutes. The dissolved clots in the cisterns were then washed out, and the remaining subarachnoid clots were aspirated as thoroughly as possible. Drainage catheters were inserted into the prepontine cistern and distal portion of the sylvian fissure. Then a bolus injection of tPA $(0.4 \mathrm{mg})$ was administered through the drainage catheters inserted into the prepontine cistern. Only one catheter was inserted into the prepontine cistern in the case of the subtemporal approach for the basilar tip aneurysms. After the closure of the dura, cisterns were washed completely with saline through the cisternal catheters.

\section{Postoperative procedures}

The catheters were left in the cisterns until 14 days after the surgery (day 14). Patients were kept in the normotensive and normovolemic state by measuring blood pressure, body weight, water balance, blood laboratory data, and central venous pressure. Induced hypertension and hypervolemic therapy was given only in the three patients who developed symptomatic vasospasm. The efficacy of the new protocol against cerebral vasospasm was assessed by the occurrence of symptomatic vasospasm and new areas of low density on CT. The occurrence of symptomatic vasospasm was evaluated by the presence of newly developed neurological deterioration (delayed ischemic neurological deficit). CT was performed immediately after the operation, on the next day, and three times a week or when delayed ischemic neurological deficit occurred. The final outcome was evaluated according to the Glasgow Outcome Scale at 1 month after the surgery. ${ }^{11)}$

\section{Results}

The preoperative evaluation found 49 patients in Hunt and Kosnik grade II, 16 in grade III, and five in grade IV (Table 1). The dose of tPA required to wash out the subarachnoid clots was $1.6 \mathrm{mg}$ in 55 patients and $3.2 \mathrm{mg}$ in 15 patients. Four of the 55 patients treated with $1.6 \mathrm{mg}$ tPA required additional injection of tPA through the catheters, once $(0.8 \mathrm{mg})$ in two cases and twice $(0.8 \mathrm{mg} \times 2)$ in two patients.

Symptomatic vasospasm occurred in three patients (Table 2). Two patients with left middle cerebral artery aneurysm (preoperative Hunt and Kosnik grade II) developed motor aphasia on day 10 and day 12. One patient with left internal carotidposterior communicating artery aneurysm showed hemiparesis from day 5 . These patients underwent induced hypertension and hypervolemic therapy. The motor aphasia completely resolved in the 
Table 1 Overall outcome

\begin{tabular}{|c|c|c|c|c|c|c|}
\hline $\begin{array}{l}\text { Preoperative } \\
\text { H\&K grade }\end{array}$ & \multicolumn{5}{|c|}{ Postoperative outcome* } & Total \\
\hline II & $41(83.7 \%)$ & $5(10.2 \%)$ & $1(2.0 \%)$ & 0 & $2(4.1 \%)$ & 49 (100\%) \\
\hline III & $13(81.3 \%)$ & $1(6.3 \%)$ & $1(6.3 \%)$ & 0 & $1(6.3 \%)$ & 16 (100\%) \\
\hline IV & $1(20.0 \%)$ & $2(40.0 \%)$ & $2(40.0 \%)$ & 0 & 0 & $5(100 \%)$ \\
\hline Total & $55(78.6 \%)$ & $8(11.4 \%)$ & $4(5.7 \%)$ & 0 & $3(4.3 \%)$ & 70 (100\%) \\
\hline
\end{tabular}

${ }^{*}$ According to the Glasgow Outcome Scale: GR, good recovery; MD, moderately disabled; SD, severely disabled; PVS, persistent vegetative state; D, dead. H\&K: Hunt and Kosnik.

Table 2 Summary of three patients with symptomatic vasospasm

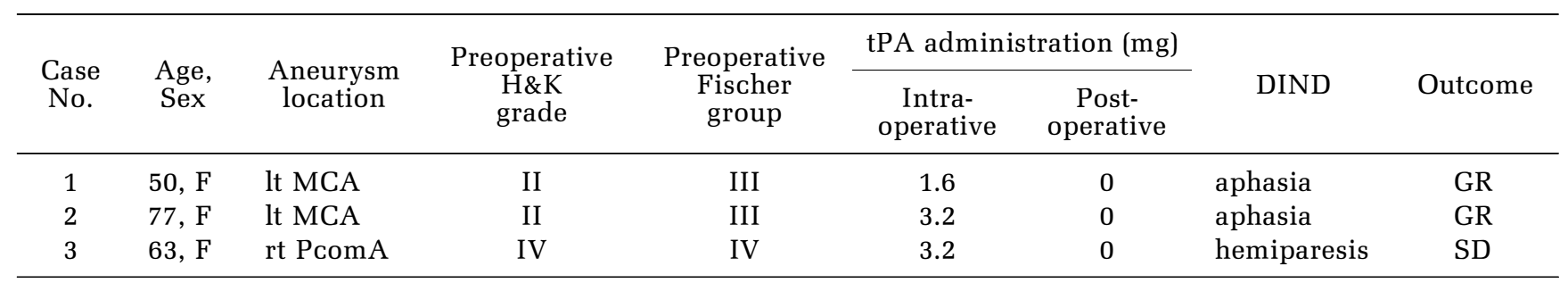

DIND: delayed ischemic neurological deficit, GR: good recovery, H\&K: Hunt and Kosnik, MCA: middle cerebral artery, PcomA: posterior communicating artery, SD: severely disabled, tPA: tissue-type plasminogen activator.

former two patients but the hemiparesis could not be fully improved in the latter patient. Two patients, one with aphasia and one with hemiparesis, suffered cerebral vasospasm which caused cerebral infarction defined as a low density area on CT (Figs. 1-3).

The outcome at 1 month postoperatively is shown in Table 1. Fifty-five patients $(78.6 \%)$ had good recovery, eight $(11.4 \%)$ had moderate disability, four $(5.7 \%)$ had severe disability, and three $(4.3 \%)$ died. None of the deaths was due to vasospasm. Two patients suffered rerupture of the aneurysm following incomplete clipping and one patient died of myocardial infarction.

Hemorrhagic complications occurred in two patients, one patient suffered SAH in the sylvian fissure on day 3 and the other patient developed an epidural hematoma on day 6. tPA was only administered during surgery in both cases, and not through the catheter following surgery. Neither patient showed neurological deficits due to the hemorrhage and surgical treatment was not necessary.

\section{Discussion}

\section{Intraoperative bolus injection of tPA}

Injection of tPA into the cisterns is a clinically effective method for preventing vasospasm, based on detailed experimental work. ${ }^{3-5,7,22)}$ A relatively large dose (7.5-15 mg) of tPA administered to the exposed cisterns immediately before completion of the surgery prevented vasospasm in 14 of 15 patients. ${ }^{6)}$ The treatment failed to clear the clots in only one patient who developed severe vasospasm. Injection of 3,10 , or $13 \mathrm{mg}$ of $\mathrm{tPA}$ into the cistern at the end of the operation in 30 patients showed that the patients receiving $13 \mathrm{mg}$ tPA had fewer blood clots on the first postoperative CT scan and less angiographic vasospasm. ${ }^{18)}$ Although this simple method appeared effective, the results of the randomized study of intraoperative tPA injection failed to confirm the prevention of vasospasm. ${ }^{2)}$

\section{Postoperative injection of tPA and cisternal drainage}

Postoperative continuous cisternal drainage to remove clots and breakdown products from the cisterns is widely used in Japan. ${ }^{10,12,15,16)}$ Cisternal irrigation using urokinase, an early precursor of intrathecal fibrinolytic therapy, ${ }^{33)}$ is also common in Japan, where a policy of early surgery has been actively pursued. Recently, a low frequency of symptomatic vasospasm after SAH $(10.7 \%)$ was reported in patients treated by urokinase cisternal irrigation therapy. ${ }^{19)}$ We previously described postoperative cisternal tPA injection for prevention of vasospasm. Combined postoperative multiple intrathecal injection of tPA (a total of $2 \mathrm{mg}$ of tPA 


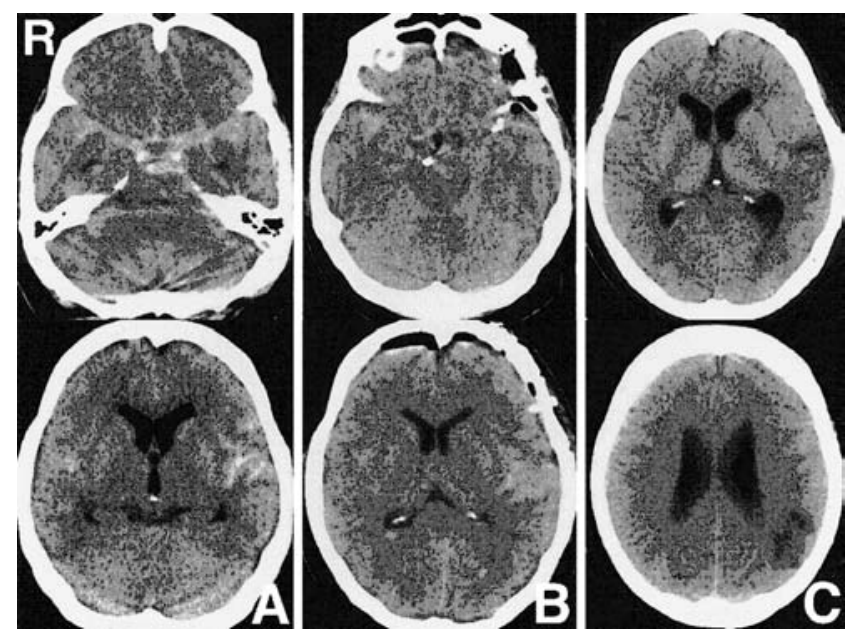

Fig. 1 Serial computed tomography (CT) scans in a 50-year-old female with symptomatic vasospasm due to ruptured left middle cerebral artery aneurysm (Case 1 in Table 2). Preoperative CT scans (A) were obtained on admission within 24 hours after subarachnoid hemorrhage (SAH), postoperative CT scans (B) immediately after the operation, and follow up CT scans (C) 1 month after SAH. Postoperative CT scans showed clearance of the SAH, but she suffered transient motor aphasia, which was completely resolved by induced hypertension and hypervolemic therapy. Followup CT scans showed vasospasm had caused cerebral infarction in the left parietal cortex.

per day) with cisternal drainage resulted in no case of symptomatic vasospasm in patients with thick subarachnoid clots. ${ }^{16)}$ Only $13 \%$ of patients showed moderate $(10 \%)$ and severe $(3 \%)$ angiographical vasospasm. However, the risk of infections caused by the injection of tPA through the cisternal catheters cannot be excluded, although the incidence is nearly zero in our practice. Therefore, we examined the effectiveness of intraoperative irrigation of tPA.

\section{Hemorrhagic complications of tPA treatment}

The most important issue with tPA treatment is the risk of hemorrhagic complication. The bleeding risk apparently depends on the dose of tPA and the method of tPA administration. A series of 15 patients treated with bolus injection of tPA resulted in one large epidural hematoma clearly related to the high dose of tPA $(15 \mathrm{mg}){ }^{6}{ }^{6}$ In addition, a series of 30 patients treated with 3,10 , or $13 \mathrm{mg}$ of tPA included two bleeding complications (one intracerebral

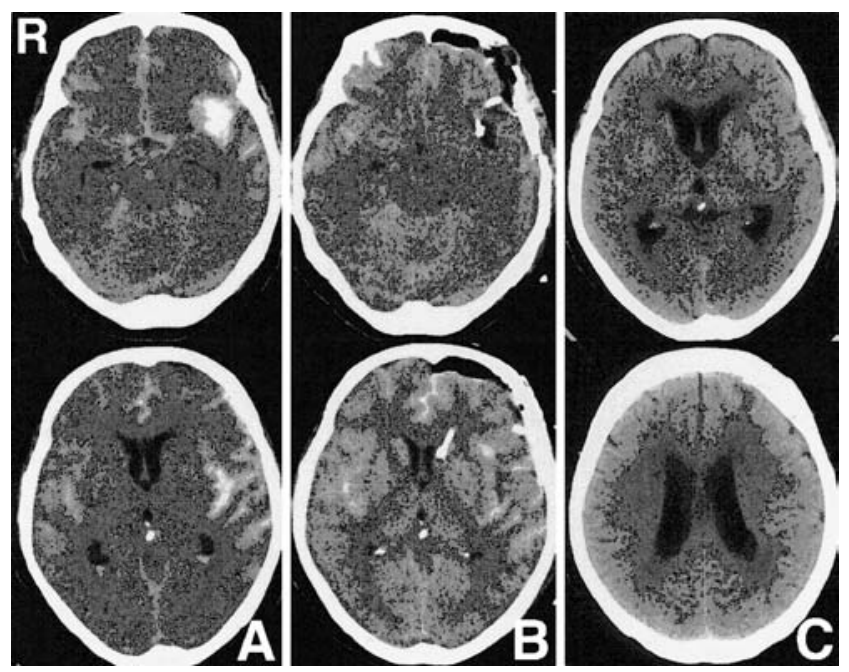

Fig. 2 Serial computed tomography (CT) scans in a 77-year-old female with symptomatic vasospasm due to ruptured left middle cerebral artery aneurysm (Case 2 in Table 2). Preoperative CT scans (A) were obtained on admission within 24 hours after subarachnoid hemorrhage (SAH), postoperative CT scans (B) immediately after the operation, and follow-up CT scans (C) 1 month after SAH. Postoperative CT scans showed clearance of the SAH, but she developed motor aphasia, which was completely resolved by induced hypertension and hypervolemic therapy. Follow-up CT scans showed no cerebral infarction.

hemorrhage in the $3 \mathrm{mg}$ group and one epidural hematoma in the $10 \mathrm{mg}$ group). ${ }^{18)}$ However, bleeding complications were absent among 20 patients treated with $10 \mathrm{mg}$ of tPA. ${ }^{25)}$ Furthermore, a randomized trial with $10 \mathrm{mg}$ of tPA revealed no significant difference in the incidence of bleeding complications between the placebo and tPA groups. ${ }^{21)}$

Three studies with multiple postoperative injections of tPA including our previous study (single injection dose: $0.5 \mathrm{mg}, 2 \mathrm{mg}, 0.042$ to $1 \mathrm{mg}$, respectively) confirmed the absence of bleeding complications due to tPA. ${ }^{16,31,34)}$ However, a multicenter trial with tPA administered via a catheter left in the cistern $(0.1,0.2$, or $0.4 \mathrm{mg}$, three times daily) found that four of 53 patients experienced hemorrhagic complications, including one case of $\mathrm{SAH}$ in the $0.1 \mathrm{mg}$ group, two cases of SAH in the $0.2 \mathrm{mg}$ group, and one case of epidural hematoma. ${ }^{20)}$ Taken together, the bleeding risk of tPA treatment appears to be acceptable either using an intraoperative bolus injection or multiple postoperative injections.

The present method of intraoperative cisternal 


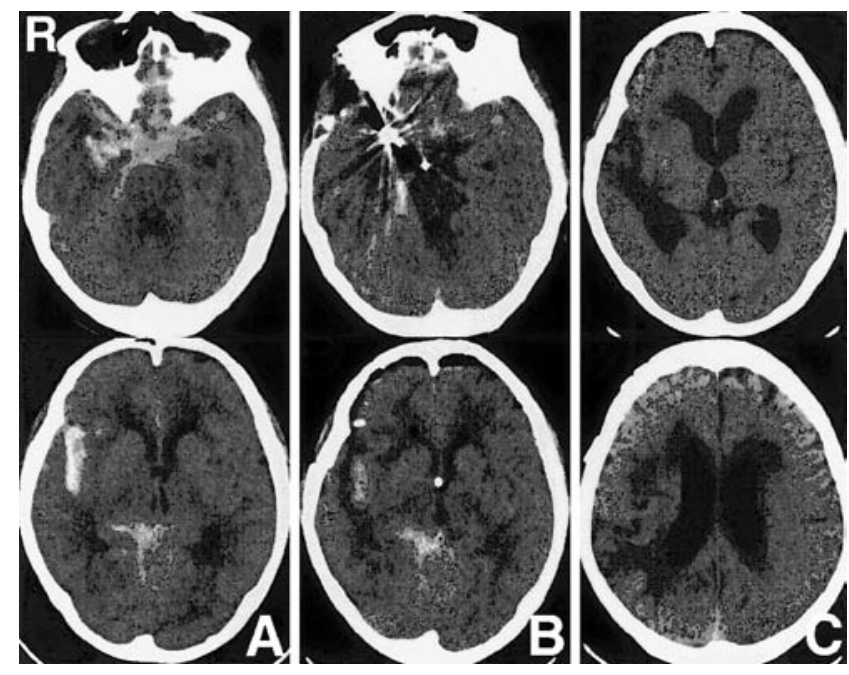

Fig. 3 Serial computed tomography (CT) scans in a 63-year-old female with symptomatic vasospasm due to ruptured right posterior communicating artery aneurysm (Case 3 in Table 2). Preoperative CT scans (A) were obtained on admission within 24 hours after subarachnoid hemorrhage (SAH), postoperative CT scans (B) immediately after the operation, and follow-up CT scans (C) 1 month after SAH. Postoperative CT scans showed clearance of the SAH, but she showed left severe hemiparesis, which was not completely improved by induced hypertension and hypervolemic therapy. Followup CT scans showed vasospasm had caused multiple cerebral infarctions. Her severe hemiparesis persisted at discharge.

irrigation with tPA was modified from our previous method with multiple postoperative injections of tPA to try to minimize the risk of bleeding complications. This series of 70 patients suffered no hemorrhagic complication due to tPA treatment.

\section{Efficacy of clot clearance}

The present study also demonstrated the efficacy of clot clearance by intraoperative cisternal irrigation with 1.6 or $3.2 \mathrm{mg}$ of tPA. Clot resolution was confirmed by direct observation of the exposed cistern and by CT following surgery. Four patients receiving a $1.6 \mathrm{mg}$ dose of tPA had areas of high density of more than $65 \mathrm{HU}$ on CT within 24 hours of surgery. Additional injections of tPA through the cisternal catheter were effective to dissolve the residual clots, as suggested by our previous findings. The present study suggests that $3.2 \mathrm{mg}$ is the optimum dose of tPA for intraoperative cisternal irrigation therapy.
The present intraoperative tPA irrigation procedure combines intraoperative injection of tPA and postoperative cisternal drainage. The present method differs from previous procedures using an intraoperative bolus injection of tPA, because the clots dissolved by tPA can be evacuated by intraoperative washing or drainage through the catheter (Table 3). The previous method of bolus injection administered a single dose (7.5-15 $\mathrm{mg}$ ) of tPA in the exposed cisterns immediately before completion of the surgery. ${ }^{2,6,18)}$ Therefore, the dissolved clots remained within the cerebrospinal fluid for a long time after surgery. Although the pathogenesis of delayed vasospasm after SAH is not fully understood, hemoglobin, superoxides, free radicals, lipid peroxides, or prostanoids generated during subarachnoid clot lysis are all considered to be potent spasmogenic substances. ${ }^{1,13,21,22)}$ Therefore, immediate drainage of the dissolved clots is expected to prevent vasospasm following $\mathrm{SAH}$.

\section{Prevention of vasospasm}

Symptomatic vasospasm occurred in three of our 70 consecutive patients $(4.3 \%)$. Our previous study using multiple postoperative injections of tPA through cisternal catheters resulted in no cases of symptomatic vasospasm among 30 patients. ${ }^{16)}$ However, the control group contained 11 cases of symptomatic vasospasm (15\%) among 75 patients. The rate of symptomatic vasospasm in the present study is also low compared to other series, such as that using continuous urokinase cisternal irrigation therapy resulting in three cases of symptomatic vasospasm $(10.7 \%)$ among 28 patients. ${ }^{19)}$

The three patients with symptomatic vasospasm in the present study showed relatively good clearance of clots by CT following surgery. The side of the symptomatic vasospasm was identical to the aneurysm and craniotomy side in all cases. Therefore, surgical maneuver or environmental changes due to rupture of the aneurysm including brain tissue damage may have caused the vasospasm. In addition, the general physiological conditions, including hypovolemia, hyponatremia, etc., are also significantly related to the occurrence of vasospasm, although the physiological data of our three patients were within the normal ranges.

\section{Conclusion}

The present method of intraoperative cisternal irrigation of tPA with cisternal drainage is safe and effective for the prevention of symptomatic vasospasm following $\mathrm{SAH}$, and reduces the risk of postoperative infection and/or hemorrhage due to injection through a catheter. 
Table 3 Studies of tissue-type plasminogen activator (tPA) for the prevention of vasospasm after subarachnoid hemorrhage

\begin{tabular}{|c|c|c|c|c|c|c|c|}
\hline Author (Year) & $\begin{array}{l}\text { No. of } \\
\text { patients }\end{array}$ & $\begin{array}{l}\text { Dose of } \\
\text { tPA (mg) }\end{array}$ & $\begin{array}{l}\text { Intra- } \\
\text { operative } \\
\text { use }\end{array}$ & $\begin{array}{l}\text { Post- } \\
\text { operative } \\
\text { use }\end{array}$ & $\begin{array}{l}\text { Cisternal } \\
\text { drainage }\end{array}$ & DIND & $\begin{array}{c}\text { Hemorrhagic } \\
\text { complica- } \\
\text { tions }\end{array}$ \\
\hline \multirow{2}{*}{ Findlay et al. (1991) ${ }^{6)}$} & 5 & 15 & + & - & - & $1 / 5(20 \%)$ & $2 / 5(40 \%)$ \\
\hline & 1 & 7.5 & + & - & - & 0 & 0 \\
\hline \multirow[t]{2}{*}{ Öhman et al. $(1991)^{18)}$} & 10 & 13 & + & - & - & $1 / 10(10 \%)$ & 0 \\
\hline & 10 & 10 & + & - & - & 0 & $1 / 10(10 \%)$ \\
\hline \multirow{2}{*}{ Zabramski et al. (1991) ${ }^{34)}$} & 4 & 5 & - & + & $-{ }^{*}$ & 0 & $4 / 4(100 \%)$ \\
\hline & 5 & $0.5 \times 3$ & - & + & $-*$ & 0 & 0 \\
\hline Stolke and Seifert $(1992)^{25)}$ & 20 & 10 & + & - & - & $1 / 20(5 \%)$ & 0 \\
\hline Mizoi et al. $(1993)^{16)}$ & 30 & $2-26$ & - & + & + & 0 & $3 / 30(10 \%)$ \\
\hline Seifert et al. $(1994)^{23)}$ & 52 & 10 & + & - & - & $4 / 52(8 \%)$ & 0 \\
\hline Present series & 70 & $1.6-3.2$ & + & $+* * *$ & + & $3 / 70(4 \%)$ & $2 / 70(3 \%)$ \\
\hline
\end{tabular}

${ }^{*}$ Cisternal catheter was used for the tPA injection. ${ }^{* *}$ Each dose of tPA was administered three times daily for 5 days.

${ }^{* * *}$ tPA was injected through the cisternal catheters in four of 55 patients in the $1.6 \mathrm{mg}$ tPA group. DIND: delayed ischemic neurological deficits.

\section{References}

1) Asano T, Takakura K, Sano K, Kikuchi H, Nagai H, Saito I, Tamura A, Ochiai C, Sasaki T: Effects of a hydroxyl radical scavenger on delayed ischemic neurological deficits following aneurysmal subarachnoid hemorrhage: results of a multicenter, placebo-controlled double blind trial. J Neurosurg 84: 792-803, 1996

2) Findlay JM, Kassell NF, Weir BKA, Haley EC Jr, Kongable G, Germanson T, Truskowski L, Alves WM, Holness RO, Knuckey NW, Yonas H, Steinberg GK, West M, Winn HR, Ferguson G: A randomized trial of intraoperative, cisternal tissue plasminogen activator for prevention of vasospasm. Neurosurgery 37: 168-178, 1995

3) Findlay JM, Weir BKA, Gordon P, Grace M, Baughman R: Safety and efficacy of intrathecal thrombolytic therapy in a primate model of cerebral vasospasm. Neurosurgery 24: 491-498, 1989

4) Findlay JM, Weir BKA, Kanamaru K, Grace M, Gaughman R: The effect of timing of intrathecal fibrinolytic therapy on cerebral vasospasm in a primate model of subarachnoid hemorrhage. Neurosurgery 26: 201-206, 1990

5) Findlay JM, Weir BKA, Kanamaru K, Grace M, Gordon P, Gaughman R, Howarth A: Intrathecal fibrinolytic therapy after subarachnoid hemorrhage: dosage study in a primate model and review of literature. Can J Neurol Sci 16: 28-40, 1989

6) Findlay JM, Weir BKA, Kassell NF, Disney LB, Grace
MG: Intracisternal recombinant tissue plasminogen activator after aneurysmal subarachnoid hemorrhage. J Neurosurg 75: 181-188, 1991

7) Findlay JM, Weir BKA, Steinke D, Tanabe T, Gordon P, Grace M: Effect of intrathecal thrombolytic therapy on subarachnoid clot and chronic vasospasm in a primate model of SAH. J Neurosurg 69: 723-735, 1988

8) Fisher CM, Kistler JP, Davis JM: Relation of cerebral vasospasm to subarachnoid hemorrhage visualized by computerized tomographic scanning. Neurosurgery 6: 1-9, 1980

9) Inagawa $\mathrm{T}$, Yamamoto $\mathrm{H}$, Kamiya $\mathrm{K}$ : Effect of clot removal on cerebral vasospasm. J Neurosurg 72: 224-230, 1990

10) Ito U, Tomita H, Yamazaki S, Takada $Y$, Inaba $Y$ : Enhanced cisternal drainage and cerebral vasospasm in early aneurysm surgery. Acta Neurochir (Wien) 80: 18-23, 1986

11) Jennett B, Bond $M$ : Assessment of outcome after severe brain damage. Lancet 1: 480-484, 1975

12) Kamii H, Kato H, Kinouchi H, Chan PH, Epstein CJ, Akabane A, Okamoto H, Yoshimoto T: Amelioration of vasospasm after subarachnoid hemorrhage in transgenic mice overexpressing CuZn-superoxide dismutase. Stroke 30: 867-871, 1999

13) Kawakami Y, Shimamura Y: Cisternal drainage after early operation of ruptured intracranial aneurysm. Neurosurgery 20: 8-14, 1986

14) Ljunggren B, Saveland H, Brandt L, Zygmunt S: Early operation and overall outcome in aneurysmal subarachnoid hemorrhage. J Neurosurg 62: 547-551, 
1985

15) Mizoi K, Yoshimoto T, Fujiwara S, Sugawara $T$, Takahashi A, Koshu K: Prevention of vasospasm by clot removal and intrathecal bolus injection of tissue-type plasminogen activator. Neurosurgery 28: 807-813, 1991

16) Mizoi K, Yoshimoto $T$, Takahashi A, Fujiwara S, Koshu K, Sugawara T: Prospective study on the prevention of cerebral vasospasm by intrathecal fibrinolytic therapy with tissue-type plasminogen activator. J Neurosurg 78: 430-437, 1993

17) Mizukami M, Kawase T, Usami T, Tazawa T: Prevention of vasospasm by early operation with removal of subarachnoid blood. Neurosurgery 10: 301-307, 1981

18) Ohman J, Servo A, Heiskanen O: Effect of intrathecal fibrinolytic therapy on clot lysis and vasospasm in patients with aneurysmal subarachnoid hemorrhage. J Neurosurg 75: 197-201, 1991

19) Sasaki T, Kodama N, Kawakami M, Sato M, Asari J, Sakurai Y, Watanabe K, Onuma T, Matsuda T: Urokinase cisternal irrigation therapy for prevention of symptomatic vasospasm after aneurysmal subarachnoid hemorrhage: a study of urokinase concentration and the fibrinolytic system. Stroke 31: 1256-1262, 2000

20) Sasaki T, Ohta T, Kikuchi H, Takakura K, Usui M, Ohnishi H, Kondo A, Tanabe H, Nakamura J, Yamada K, Kobayashi N, Ohashi Y: A phase II clinical trial of recombinant tissue-type plasminogen activator against cerebral vasospasm after aneurysmal subarachnoid hemorrhage. Neurosurgery 35: 597-605, 1994

21) Sasaki T, Wakai S, Asano T, Watanabe T, Kirino T, Sano K: The effect of a lipid hydroperoxide of arachidonic acid on the canine basilar artery. An experimental study on cerebral vasospasm. J Neurosurg 54: 357-365, 1981

22) Seifert V, Eisert WG, Stolke D, Goetz C: Efficacy of single intracisternal bolus injection of recombinant tissue plasminogen activator to prevent delayed cerebral vasospasm after experimental subarachnoid hemorrhage. Neurosurgery 25: 590-598, 1989

23) Seifert V, Stolke D, Zimmermann M, Feldges A: Prevention of delayed ischemic deficits after aneurysmal subarachnoid haemorrhage by intrathecal bolus injection of tissue type plasminogen activator (tPA). Acta Neurochir (Wien) 128: 137-143, 1994

24) Steinberg GK, Vanefsky MA, Marks MP, Adler JR Jr, Koenig GH: Failure of intracisternal tissue plasminogen activator to prevent vasospasm in certain patients with aneurysmal subarachnoid hemorrhage. Neurosurgery 34: 809-814, 1994
25) Stolke D, Seifert V: Single intracisternal bolus of recombinant tissue plasminogen activator in patients with aneurysmal subarachnoid hemorrhage. Preliminary assessment of efficacy and safety in an open clinical study. Neurosurgery 30: 877-881, 1992

26) Suzuki J, Kodama N, Yoshimoto T, Mizoi K: Ultraearly surgery of intracranial aneurysms. Acta Neurochir (Wien) 63: 185-191, 1982

27) Suzuki J, Komatsu S, Sato T, Sakurai Y: Correlation between CT findings and subsequent development of cerebral infarction due to vasospasm in subarachnoid hemorrhage. Acta Neurochir (Wien) 55: 63-70, 1980

28) Suzuki J, Mizoi K, Kayama T: Bifrontal interhemispheric approach to aneurysms of the anterior communicating artery. J Neurosurg 64: 183-190, 1986

29) Suzuki J, Onuma T, Yoshimoto T: Results of early operations on cerebral aneurysms. Surg Neurol 11: 407-412, 1979

30) Suzuki J, Yoshimoto T, Kayama T: Surgical treatment of middle cerebral artery aneurysms. J Neurosurg 61: 17-23, 1984

31) Taneda M: Effect of early operation for ruptured aneurysms on prevention of delayed ischemic symptoms. J Neurosurg 57: 622-628, 1982

32) Usui M, Saito N, Hoya K, Todo T: Vasospasm prevention with postoperative intrathecal thrombolytic therapy: A retrospective comparison of urokinase, tissue plasminogen activator, and cisternal drainage alone. Neurosurgery 34: 235-245, 1994

33) YoshidaY, Ueki, S, Takahashi A, Takagi H, Torigoe $\mathrm{H}$, Kudo S: [Intrathecal irrigation with urokinase in ruptured cerebral aneurysm cases. Basic study and clinical application]. Neurol Med Chir (Tokyo) 25: 989-997, 1985 (Jpn, with Eng abstract)

34) Zabramski JM, Spetzler RF, Lee KS, Papadopoulos SM, Bovill E, Zimmerman RS, Bederson JB: Phase I trial of tissue plasminogen activator for the prevention of vasospasm in patients with aneurysmal subarachnoid hemorrhage. J Neurosurg 75: 189-196, 1991

Address reprint requests to: H. Kinouchi, M.D., Department of Neurosurgery, Akita University School of Medicine, 1-1-1 Hondo, Akita 010-8543, Japan. e-mail: kinouchi@nsg.med.akita-u.ac.jp

Commentary on this paper appears on the next page. 


\section{Commentary}

This article reports a very relevant experience with a large cohort of patients treated with a new protocol of intracisternal rTPA in conjunction with craniotomy for aneurysm clipping, in high Fisher grade patients, with the aim of preventing symptomatic vasospasm.

As the authors discuss, the rationale of thrombolysis for vasospasm prevention is based on a rigorous experimental foundation, including mechanistic studies and highly compelling results in primates by Findlay and Weir's group and others more than a decade ago. Human trials were plagued with concern for hemorrhagic post-craniotomy complications in high dose rTPA groups, and lesser effectiveness at lower doses. Ultimately, this line of therapy was largely abandoned, as even a single fatal or disabling hemorrhage would not be tolerated in an otherwise prophylactic regimen where most patients would be expected to do well without this therapy. And as we all know, only a small fraction of patients, even with high Fisher grade, eventually develop serious complications from vasospasm.

Since that experience, there has been a resurgence of interest in rTPA for intraventricular hemorrhage, with highly promising results in ongoing trials, and a newly funded trial in North America (MISTIE trial) for thrombolytic aspiration of intracerebral hematoma. In those experiences, smaller doses of rTPA are proposed, with little added significant hemorrhagic risk, and potentially outweighed by the serious consequences of those types of bleeds where most patients die or are severely disabled. So it is cogent to revisit the question whether intracisternal or even intraventricular rTPA in aneurysmal subarachnoid hemorrhage would be safe at lower doses, and would prevent symptomatic vasospasm.

Kinouchi and colleagues propose such a protocol, carefully developed at their center. And they show convincing safety, without a single bleed or infection in a large prospective cohort. This is excellent Phase I data, warranting further investigation of this protocol. But while they make some claims regarding prevalence of vasospasm or clinical outcomes, these cannot be accepted without any form of control. It is not clear whether the safe low doses proposed in this study added any significant benefit in terms of prevalence or outcome from vasospasm, and what might be the results of placebo (non rTPA irrigation) and rTPA dose escalation with this new protocol. Historical controls cannot be accepted, as similar ranges of outcome have been reported with other neurocritical care measures of aggressive hydration, ventricular drainage without thrombolysis, calcium channel blockers, and/or transcranial Doppler surveillance and aggressive endovascular intervention for symptomatic spasm.

What is now needed is a Phase II study with placebo, dose escalation and blinding. If safety is maintained and biologic effects confirmed at a safe optimized dose, a Phase III study could be conducted using the optimized dosage, with third party blinded adjudication of outcome, and randomized control for other treatment adjuncts and potential biases. The authors have the experience and case volume to accomplish these important studies in upcoming years, and finally accredit or discard this important therapeutic modality.

Issam A. AWAD, M.D., M.Sc., F.A.C.S., M.A. (Hon.) Professor of Neurosurgery Evanston Northwestern Healthcare Northwestern University Feinberg School of Medicine Evanston, Illinois, U.S.A.

The authors report 70 patients with Fisher grade III or IV SAH undergoing early aneurysm surgery, and mainly intraoperative instillation of relatively small doses of tPA. Only two patients developed hemorrhagic complications, neither needing surgery, and there were only three cases of delayed ischemia presumed due to vasospasm, two of which were reversible. No infections were reported in spite of cisternal catheters left in place on continuous drainage for two weeks. Overall outcome was good for an unselected group with significant hemorrhage, particularly for assessment at one month after operation.

This study is interesting in view of the low incidence of delayed ischemia compared with many of the previous reports (which have been summarized in a useful table) and in view of the low dose of tPA used. Why is this? Perhaps the answer lies in the technique used, of opening the basal cisterns widely at operation, instilling the tPA then waiting for over 30 minutes, washing out the dissolved clot and removing as much as possible of any remaining clot, and finally instilling a little more tPA and washing the cisterns again after dural closure. With good surgical technique, the risk of causing further vascular or other damage should be small, although operating time may be considerably more. As discussed, the risk of causing infection by multiple postoperative cisternal injections is also much less, and one would expect also less risk of hemorrhagic complications. Judged by postoperative CT scanning this procedure seemed very effective, with only four patients needing further $t P A$.

It would perhaps be difficult to justify, but would be very interesting to see the results of a randomized control study where one group received the same 
cisternal opening and washout but no fibrinolytic instillation.

Nicholas W. C. Dorsch, M.D., F.R.C.S., F.R.A.C.S. Department of Neurosurgery Westmead Hospital Sydney, Australia

Removal of subarachnoidal clot by inducing fibrinolysis with intracisternal administration of tissue-type plasminogen activator (tPA) and subsequent drainage is thought to be an effective clinical manipulation to prevent vasospasm after aneurysmal subarachnoid hemorrhage (SAH). Various methods of administration and dosages of tPA have been tried, and different therapeutic results were obtained.

In this article, the authors demonstrated that a single intraoperative administration of small dose (1.6-3.2 mg) tPA to the exposed cistern with continuous cisternal drainage could reduce the incidence and severity of symptomatic vasospasm in patients with high Fisher grade SAH after successful clipping of the ruptured aneurysm. The risk of hemorrhagic complication is also lower than in the other reported series.

It is generally accepted that intra-cisternal injection of tPA with proper drainage of the dissolved clot could be a good prophylaxis for vasospasm after aneurysmal SAH. However, the optimal dosage of tPA to effectively dissolve the subarachnoid clot and minimize the risk of bleeding complication has not yet clearly defined. The present article provides the message that intra-cisternal injection of a single and relatively small dose of tPA has a significant therapeutic effect. A large-scale clinical trial to define the optimal dose and the best method of administration in the future is very important for the standardization of this therapeutic modality.

Yong-Kwang TU, M.D. Department of Neurosurgery College of Medicine and Hospital National Taiwan University Taipei, Taiwan, R.O.C. 